\title{
Rayonnement $X$ mou produit à l'aide d'électrons relativistes traversant des multicouches
}

\author{
J.-M. André, R. Barchewitz et C. Bonnelle \\ Laboratoire de Chimie Physique, Matière et Rayonnement, URA 176 du CNRS, Université \\ Pierre et Marie Curie, 11 rue Pierre et Marie Curie, 75231 Paris cedex 05, France
}

\begin{abstract}
Nous avons calculé les distributions spectrale et spatiale du rayonnement émis lorsque des électrons relativistes traversent une structure périodique multicouche de période nanométrique dans des conditions d'incidence voisines des conditions de Bragg. Ce rayonnement est situé dans le domaine $\mathrm{X}$ mou; il est compris dans un étroit domaine d'énergie et son intensité est supérieure à celle d'un tube à $R X$ conventionnel fonctionnant dans le même domaine spectral.
\end{abstract}

Lorsqu'une particule chargée se déplace selon un mouvement uniforme dans un milieu matériel optiquement hétérogène, c'est à dire présentant un gradient de constante diélectrique, elle y produit une onde électromagnétique dont l'intensité augmente avec la valeur du gradient. L'intensité devient importante si la particule passe à travers une interface séparant deux milieux de constante diélectrique différente (rayonnement de transition) [1].

Afin d'augmenter l'intensité et la monochromaticité de ce rayonnement, il a été proposé d'utiliser des radiateurs constitués d'empilements multicouches périodiques artificiels, d'épaisseurs calculées de telle sorte qu'il existe des interférences constructives entre les ondes émises par chacune des couches [2],[3]. L'étude théorique du rayonnement de transition émis par de tels empilements traversés perpendiculairement par une particule chargée a montré que des phénomènes particuliers doivent se produire au voisinage des conditions de Bragg [4].

Nous avons calculé l'intensité émise par une multicouche traversée par des électrons relativistes au voisinage des conditions de Bragg. Différents modèles théoriques avaient été établis pour traiter le cas d'un cristal irradié dans les conditions de Bragg [5][6]; ils avaient prévu l'existence d'un maximum étroit de la distribution spectrale émise par un tel système. Nous avons adapté le modèle de la référence 5 au cas d'une multicouche [7].

La longueur d'onde du rayonnement produit par des empilements multicouches de période nanométrique est de l'ordre du nanomètre, c'est dire du domaine des rayons $\mathrm{X}$ 
mous. Dans la géométrie que nous avons considérée, le faisceau d'électrons est horizontal et son angle d'incidence est voisin de l'angle de Bragg pour un rayonnement de $1 \mathrm{keV}$ réfléchi par une multicouche constituée de 35 paires Mo $(1,13 \mathrm{~nm}) / \mathrm{C}(2,27 \mathrm{~nm})$, soit égal à $10,5^{\circ}$. Dans ces conditions, la distribution spectrale du rayonnement présente un pic étroit de $15 \mathrm{eV}$ de large environ. Au maximum de la distribution, le nombre de photons émis par un électron de $300 \mathrm{MeV}$ traversant la multicouche serait 0,0175 / stéradian / eV. $\mathrm{Ce}$ nombre augmente quasi linéairement avec l'énergie des électrons entre 200 et $500 \mathrm{MeV}$ et diminue très rapidement en-dessous de $100 \mathrm{MeV}$.

La distribution angulaire (photons / stéradian / eV / électron) est présentée sur la figure en fonction de l'angle azimutal $\Phi$, c'est-à-dire de l'angle que fait la direction d'observation avec le plan horizontal, et en fonction de l'angle $\theta$ que fait la direction d'observation avec la direction d'incidence. Les photons sont émis dans deux directions priviligiées, situées de part et d'autre de la direction d'incidence.

D'après notre modèle, par suite de sa très forte monochromaticité, le type de source proposé émettrait un nombre de photons $10^{3}$ fois supérieur au nombre de photons émis par un tube à $\mathrm{RX}$ conventionnel par stéradian dans une raie caractéristique intense de $1 \mathrm{eV}$ de large, située au voisinage du $\mathrm{keV}$.

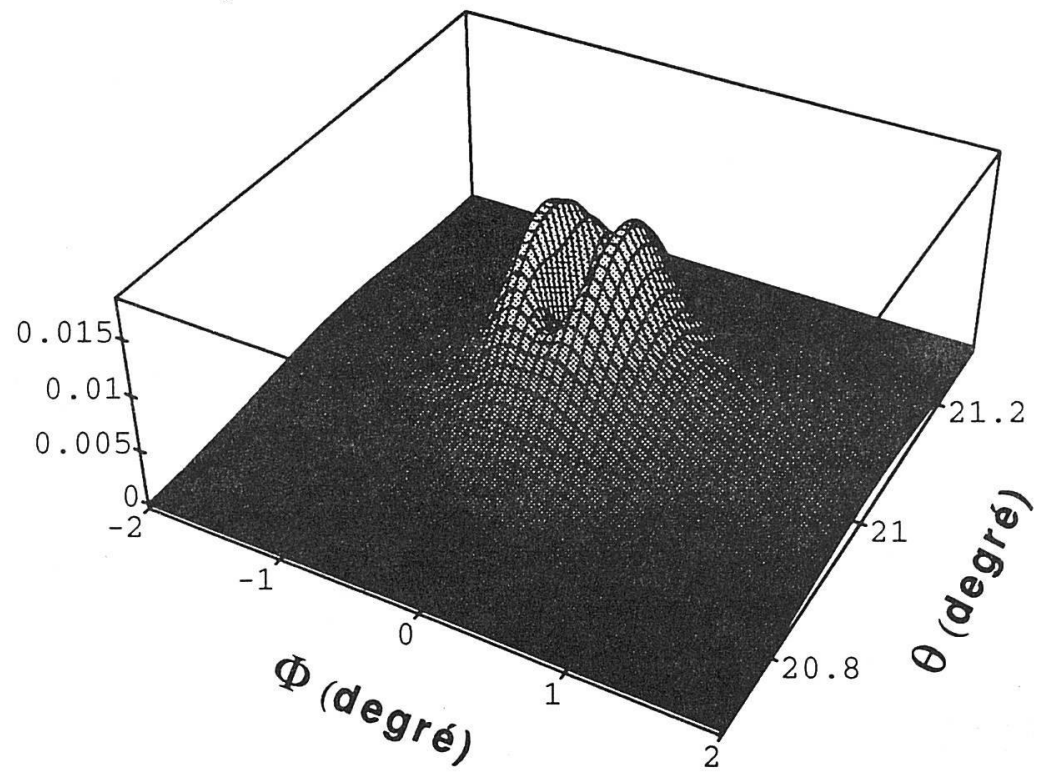

[1] Ginsburg V.L., Tsytovitch V.N., Transition radiation and transition scattering (Adam Hilger Series on Plasma Physics, Bristol, 1990).

[2] Law C.T., Kaplan A.E., Optics Lett. 12 (1987) 900.

[3] André J.-M., Barchewitz R., Bonnelle C., Pardo B., J. Optics (Paris) 24 (1993) 31.

[4] Pardo B., André J.-M., Phys. Rev. A 40 (1989) 1918.

[5] Dialetis D., Phys. Rev. A 17 (1978) 1113.

[6] Ya Dubovskaya I., Stepanov S.A., Ya Silenko A., Ulyanenkoff A.P., J.Phys. Condens. Matter 5 (1993) 7771.

[7] André J.-M., Barchewitz R., Bonnelle C., à paraitre. 